\title{
The Impact of Century-Scale Changes in the Core Magnetic Field on External Magnetic Field Contributions
}

\author{
Ingrid Cnossen ${ }^{1,2}$ (D)
}

Received: 24 February 2016 / Accepted: 7 July 2016

CC Springer Science+Business Media Dordrecht 2016

\begin{abstract}
The Earth's internal magnetic field controls to a degree the strength, geographic positioning, and structure of currents flowing in the ionosphere and magnetosphere, which produce their own (external) magnetic fields. The secular variation of the Earth's internal magnetic field can therefore lead to long-term changes in the externally produced magnetic field as well. Here we will examine this more closely. First, we obtain scaling relations to describe how the strength of magnetic perturbations associated with various different current systems in the ionosphere and magnetosphere depends on the internal magnetic field intensity. Second, we discuss how changes in the orientation of a simple dipolar magnetic field will affect the current systems. Third, we use model simulations to study how actual changes in the Earth's internal magnetic field between 1908 and 2008 have affected some of the relevant current systems. The influence of the internal magnetic field on low- to mid-latitude currents in the ionosphere is relatively well understood, while the effects on high-latitude current systems and currents in the magnetosphere still pose considerable challenges.
\end{abstract}

Keywords Magnetic field · Magnetosphere $\cdot$ Ionosphere $\cdot$ Secular variation $\cdot$ Current systems

\section{Introduction}

The Earth's core magnetic field plays an important role in the upper atmosphere. It affects the conductivity in the ionosphere, ionospheric plasma transport processes, the geographic locations of the magnetic equator and the auroral zones, and the coupling of the ionosphere-thermosphere system with the solar wind and the magnetosphere. Because of this, the Earth's internal magnetic field controls to a degree the structure and strength of external contributions to the magnetic field that arise from electrical currents flowing in the

\footnotetext{
$凶$ I. Cnossen

icnossen@gfz-potsdam.de; inos@bas.ac.uk

1 British Antarctic Survey, High Cross, Madingley Road, Cambridge, CB3 OET, UK

2 GFZ German Center for Geosciences, Telegrafenberg, 14473 Potsdam, Germany
} 
ionosphere and magnetosphere. While these currents are strongly driven by variable solar wind conditions on short timescales of minutes, hours, and days, on timescales of decades, centuries, and longer, the secular variation of the core magnetic field also has the potential to induce changes in these currents, and therefore the externally induced magnetic field. Such long-term changes in the externally induced magnetic field can be difficult to separate from decadal to centennial-scale changes in the core magnetic field itself. Yet it is important to separate the different sources in order to improve our understanding of the physical processes that generate them.

To find out how the secular variation of the core magnetic field could affect the external magnetic field, it is helpful to consider simplified cases first, separating the effects of variations in field strength and orientation in the case of a dipolar magnetic field. Several studies have derived scaling relations to describe how external magnetic field contributions depend on the main geomagnetic dipole moment intensity. Some of these were purely based on theoretical arguments (Siscoe and Chen 1975; Vogt and Glassmeier 2001; Glassmeier et al. 2004), while later studies revisited some of the theoretical scaling relations with numerical model simulations (Siscoe et al. 2002; Zieger et al. 2006a, 2006b; Cnossen et al. 2011, 2012a). In Sect. 2 we will review and synthesize results from these studies, aiming to derive the most realistic scaling relations currently available for the magnetic perturbations associated with various different current systems. The effects of the orientation and configuration of the main magnetic field on externally induced magnetic fields will be discussed in Sect. 3. These two sections form the basis to examine how more realistic main magnetic field changes, as described by the International Geomagnetic Reference Field (IGRF; Thébault et al. 2015), have affected the currents in the ionosphere and magnetosphere during the past century. This will be described in Sect. 4, largely based on modelling studies by Cnossen and Richmond (2013) and Cnossen (2014). Implications of these results for modelling the Earth's internal magnetic field are discussed in Sect. 5. We will finish with a brief summary and conclusions in Sect. 6.

\section{Effects of Dipole Moment Intensity Variations}

\subsection{Ionospheric Conductivity}

One of the most important effects of a change in dipole moment intensity for currents in the ionosphere-magnetosphere system is its effect on conductivity: the lower the dipole moment, the more freedom ions and electrons have to move, and therefore the larger the conductivity. This is expressed mathematically by the following equations for the ionospheric Pedersen and Hall conductivities (see, e.g., Richmond 1995):

$$
\begin{aligned}
& \sigma_{P}=\frac{N_{e} e}{B}\left(\frac{v_{i n} \Omega_{i}}{v_{i n}^{2}+\Omega_{i}^{2}}+\frac{v_{e n \perp} \Omega_{e}}{v_{e n \perp}^{2}+\Omega_{e}^{2}}\right) \\
& \sigma_{H}=\frac{N_{e} e}{B}\left(\frac{\Omega_{e}^{2}}{v_{e n \perp}^{2}+\Omega_{e}^{2}}-\frac{\Omega_{i}^{2}}{v_{i n}^{2}+\Omega_{i}^{2}}\right)
\end{aligned}
$$

where $\sigma_{P}=$ Pedersen conductivity, $\sigma_{H}=$ Hall conductivity, $N_{e}=$ electron number density, $e=$ magnitude of the electron charge, $B=$ magnitude of the magnetic field, $v_{i n}=$ collision frequency of ions with neutrals, $v_{e n \perp}=$ collision frequency of electrons with neutrals in the 


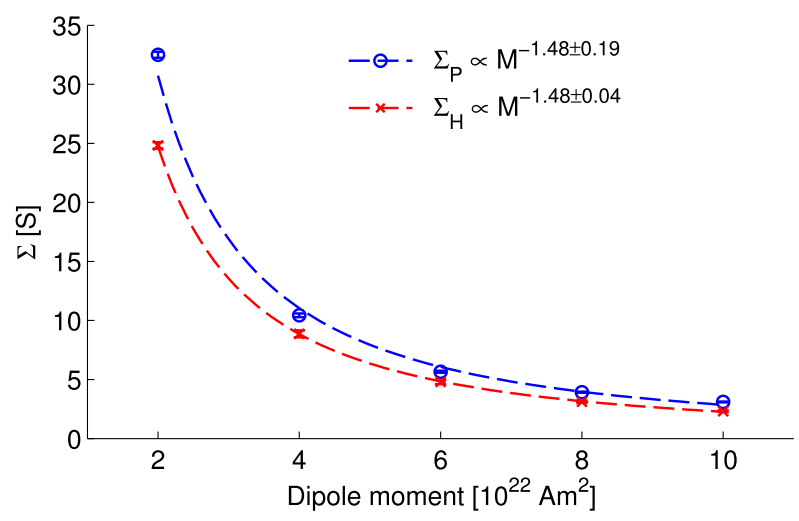

Fig. 1 Dependence of the global mean Pedersen $\left(\Sigma_{P}\right)$ and Hall $\left(\Sigma_{H}\right)$ conductance on dipole moment intensity for intermediate solar activity $(F 10.7=150 \mathrm{sfu})$ based on CMIT simulations by Cnossen et al. (2012a). Each marker represents a 24-hour average under constant solar wind conditions (Interplanetary Magnetic Field (IMF) $B_{x}=B_{y}=0 ; B_{z}=-5 \mathrm{nT}$; solar wind speed $V_{x}=-400 \mathrm{~km} / \mathrm{s} ; V_{y}=V_{z}=0$ ), with error bars indicating the standard deviation. The dashed lines indicate the fit to the indicated scaling functions. Note that the standard deviations are very small, so that the error bars are very small too and somewhat hard to see

direction perpendicular to the magnetic field, $\Omega_{i}=$ ion gyrofrequency, and $\Omega_{e}=$ electron gyrofrequency. The ion and electron gyrofrequencies are given by:

$$
\begin{aligned}
& \Omega_{i}=e B / m_{i} \\
& \Omega_{i}=e B / m_{e}
\end{aligned}
$$

where $m_{i}=$ ion mass, and $m_{e}=$ electron mass.

The magnitude of the Earth's magnetic field thus affects the Pedersen and Hall conductivities directly via $B$ appearing in the denominator of Eqs. (1a), (1b), giving larger conductivities for a weaker magnetic field. In addition, the main magnetic field strength affects the ion and electron gyrofrequencies, and thereby the heights at which these equal the ion-neutral and electron-neutral collision frequencies, which influences the vertical profile of the conductivities. A reduction in magnetic field strength causes an upward shift of the peak of the Pedersen conductivity, into a regime where the background electron densities are larger, and broadens the vertical profile of the Hall conductivity (Rishbeth 1985; Takeda 1996). The vertically integrated conductivities, i.e., the conductances, therefore also increase with a decreasing main field strength due to these effects.

Cnossen et al. (2011, 2012a) performed simulations with the Coupled MagnetosphereIonosphere-Thermosphere (CMIT) model to study how the magnetosphere, ionosphere and thermosphere respond to changes in the main magnetic field strength. The CMIT model (Wang et al. 2004, 2008; Wiltberger et al. 2004) couples the Lyon-Fedder-Mobarry (LFM) magneto-hydrodynamic (MHD) model of the magnetosphere (Lyon et al. 2004) with the Thermosphere-Ionosphere-Electrodynamics General Circulation Model (TIE-GCM; Roble et al. 1988; Richmond et al. 1992). Figure 1 illustrates how the globally averaged Pedersen and Hall conductances depend on the dipole moment intensity, $M$, based on the CMIT simulations by Cnossen et al. (2012a). Both scale approximately as $M^{-1.5}$. These results represent intermediate solar activity conditions $(F 10.7=150$ solar flux units; sfu $)$ and constant solar wind conditions with a southward directed Interplanetary Magnetic Field (IMF) of $5 \mathrm{nT}$. The scaling for the Pedersen conductance is slightly dependent on the background 
solar activity level, with the scaling being stronger for higher solar activity. This arises from an increase in the relative contribution to the Pedersen conductance from the upper part of the ionosphere, which depends more strongly on the dipole moment intensity when solar activity is higher (see Cnossen et al. (2012a) for details).

The dependence of the Pedersen and Hall conductances on the dipole moment intensity as shown in Fig. 1 is dominated by the day-side, where conductivities are generally much larger. This is exactly why models of the main magnetic field normally use night-time measurements: the generally smaller night-time conductivities tend to lead to weaker currents and smaller external magnetic field perturbations. It would therefore be useful to obtain scaling relations for the conductances separately for night-time and day-time.

An empirical relationship given by Richmond (1995) suggests that day-time values of the Pedersen and Hall conductances, $\Sigma_{P}$ and $\Sigma_{H}$, scale as:

$$
\begin{aligned}
& \Sigma_{P, \text { day }} \propto M^{-1.6} \\
& \Sigma_{H, \text { day }} \propto M^{-1.3}
\end{aligned}
$$

Glassmeier et al. (2004) obtained essentially the same scaling for the daytime Hall conductance, based on theoretical arguments and a simple Chapman layer model of ionospheric electron density. However, they suggested a considerably weaker scaling for the day-time Pedersen conductance:

$$
\Sigma_{P, \text { day }} \propto M^{-1}
$$

CMIT model simulations by Cnossen et al. (2011) suggested the following scalings of the day-time Pedersen and Hall conductances with the dipole moment intensity:

$$
\begin{aligned}
& \Sigma_{P, \text { day }} \propto M^{-1.5} \\
& \Sigma_{H, \text { day }} \propto M^{-1.7}
\end{aligned}
$$

The Pedersen conductance scaling indicated by the CMIT model (Eq. (5a)) agrees very well with the Richmond (1995) scaling (Eq. (3a)), which suggests that the Glassmeier et al. (2004) scaling (Eq. (4)) is too weak. On the other hand, the day-time Hall conductance scaling obtained from the model simulations (Eq. (5b)) is notably stronger than both the Richmond (1995) and Glassmeier et al. (2004) estimate (Eq. (3b)). Still, we suggest that the scalings of Eqs. (5a) and (5b) may be the most accurate, as the CMIT model offers a much more rigorous treatment of the electron densities than the simpler models underpinning the Richmond (1995) and Glassmeier et al. (2004) scalings. Cnossen et al. (2011) showed that, primarily because plasma transport processes depend on the magnetic field, the ionospheric electron density has a dependency on the dipole moment intensity, which further affects the ionospheric conductivities than already implied by Eqs. (1a), (1b).

At night-time, the electron density is much harder to predict than during the day, in particular at high latitudes, where it depends strongly on energetic particle precipitation from the magnetosphere. This is a much less predictable source of ionization than solar radiation, which is the dominant source of ionization during the day. This makes it more difficult to derive scaling relations for night-time conductivities, especially within the auroral zone, where particle precipitation is important. Outside of the auroral zone, conductivities are very small and do not show any dependence on the main magnetic field strength according to the simulations by Cnossen et al. (2011). Still, understanding the dependence of the conductivities within the night-time auroral zone is important, because substantial currents can flow here. 
Glassmeier et al. (2004) therefore derived a scaling relation to describe the dependence of the night-time, auroral zone conductances on dipole moment intensity based on theoretical considerations. Their scaling takes into account that changes in the size of the loss cone, which can be expected to occur for a change in dipole moment intensity, will affect energetic particle precipitation into the ionosphere, and thereby the night-time conductance. Glassmeier et al. (2004) argued that this effect can be described by multiplying a scaling for the day-time Pedersen or Hall conductances with the factor $M^{3 \gamma-3 / 2}$, where $\gamma$ takes a value between 0 and 1/2, depending on the $B_{z}$ component of the IMF. Vogt and Glassmeier (2001) suggest that $\gamma=0$ for strong northward IMF and $\gamma=1 / 2$ for strong southward IMF, while traditional scalings for magnetospheric quantities with the dipole moment intensity (e.g., Siscoe and Chen 1975) imply that $\gamma=\frac{1}{3}$. In general form, the multiplication factor suggested by Glassmeier et al. (2004), combined with the daytime conductance scalings of Eqs. (5a) and (5b), gives:

$$
\begin{aligned}
& \Sigma_{P, n i g h t} \propto M^{3(\gamma-1)} \\
& \Sigma_{H, n i g h t} \propto M^{3(\gamma-1)-0.2}
\end{aligned}
$$

These scalings suggest that the conductances in the auroral zone depend at least as strongly on the dipole moment intensity during the night as during the day (for $\gamma=1 / 2$ ), if not more strongly (for $0 \leq \gamma<1 / 2$ ). This is in contrast to the results obtained by Cnossen et al. (2011) from their CMIT model simulations, which showed very little dependence of the night-time Pedersen conductance on the dipole moment intensity, even within the auroral zone, and a dependence of the night-time Hall conductance within the auroral zone that can be approximately described as:

$$
\Sigma_{H, n i g h t} \propto M^{-0.9}
$$

This indicates a considerably weaker dependence on the dipole moment intensity than suggested by Eq. (6b), even when one assumes $\gamma=1 / 2$ (note that Cnossen et al. (2011) used IMF $B_{z}=-5 \mathrm{nT}$, which is only moderately southward). However, the version of the CMIT model used by Cnossen et al. (2011) relied on a particle precipitation parameterization that does not allow for any change in the size of the loss cone when the dipole moment intensity changes (Wiltberger et al. 2009). Without this effect, the dependence of the night-time conductances on the dipole moment intensity as found by Cnossen et al. (2011) may therefore be unrealistically weak. Here we will consider Eqs. (6a), (6b) to be the best scaling currently available for night-time conductances within the auroral zone. Zhang et al. (2015) recently developed a new approach to modelling different kinds of electron precipitation, which can be used as part of CMIT, but the implications of this new approach for the dependence of energetic particle precipitation and night-time conductivities on the dipole moment intensity have not been studied yet.

Since night-time observations are normally used to minimize the external magnetic field contributions when creating internal magnetic field models, the uncertainty regarding the potentially strong dependence of night-time conductances on the main magnetic field strength in the auroral zone is problematic for the separation of internal and external contributions to the magnetic field on long timescales. At lower latitudes, ionospheric models such as the CMIT model used by Cnossen et al. (2011, 2012a) should still give a reasonably good description of the dependence of both day- and night-time conductivities on the main magnetic field strength. 
Fig. 2 Dependence of the amplitude of the northward, eastward, and downward components of the daily variation in the Sq magnetic field at $30^{\circ}$ magnetic latitude on the dipole moment intensity for intermediate solar activity ( $F 10.7=150 \mathrm{sfu})$ based on CMIT simulations by Cnossen et al. (2012a). Each marker represents a longitudinal average, with error bars indicating the standard deviation. Dashed lines indicate the best fit to the simulation results for $M \geq 4 \times 10^{22} \mathrm{Am}^{2}$

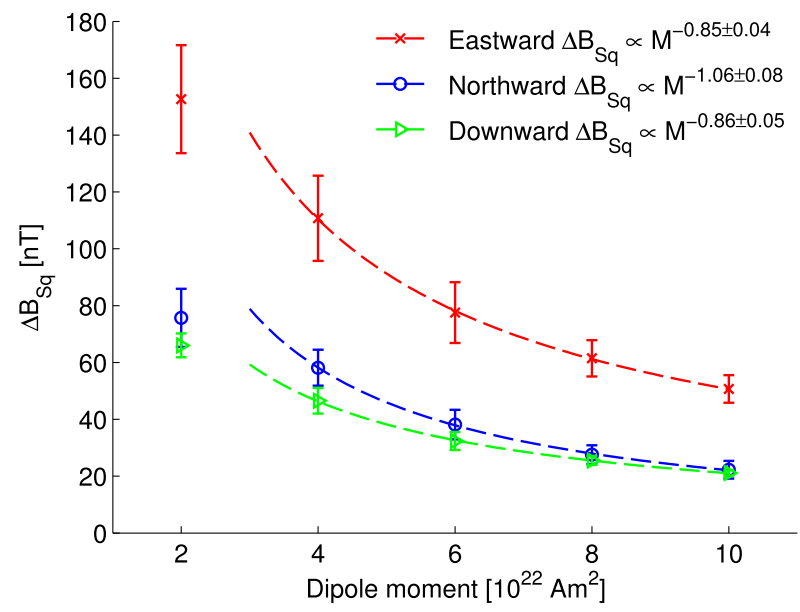

\subsection{Sq Currents and the Equatorial Electrojet}

Upper atmosphere winds, produced by the daily variation in the absorption of solar radiation, move the ionospheric plasma through the Earth's magnetic field. This acts as a dynamo, setting up currents and causing electric polarization charges and electric fields to develop. On the dayside, the ionospheric wind dynamo produces a regular current pattern, which gives a characteristic daily variation in ground magnetic perturbations at low- to mid-latitudes, known as the solar quiet (Sq) variation. The equivalent Sq current system, i.e., the horizontal currents overhead that would produce the observed magnetic perturbations on the ground, consists of a clockwise current vortex in the Southern hemisphere and counter-clockwise current vortex in the Northern hemisphere.

The Sq Hall current, $\mathbf{J}_{H}$, and Pedersen current, $\mathbf{J}_{P}$, can be written as:

$$
\begin{aligned}
& \mathbf{J}_{H}=\sigma_{H} \mathbf{b} \times\left(\mathbf{E}_{\perp}+\mathbf{v} \times \mathbf{B}\right) \\
& \mathbf{J}_{P}=\sigma_{P}\left(\mathbf{E}_{\perp}+\mathbf{v} \times \mathbf{B}\right)
\end{aligned}
$$

where $\mathbf{b}$ is a unit vector in the direction of the geomagnetic field $\mathbf{B}, \mathbf{E}_{\perp}$ is the electric field perpendicular to $\mathbf{B}$, and $\mathbf{v}$ is the wind velocity. The current associated with the $\mathbf{v} \times \mathbf{B}$ term, also known as the dynamo electric field, is to first order proportional to the product of the conductivity and the magnetic field strength (or $M$ ). If we use the scalings from Eqs. (5a) and (5b) for the daytime Pedersen and Hall conductances, this would give an approximate scaling of Sq with $M^{-0.5}$ to $M^{-0.7}$. However, the vertical shift in the Pedersen conductivity profile with a changing dipole moment intensity also changes the altitude regime that needs to be considered for the neutral winds, with winds typically being stronger at higher altitudes. Since a weaker dipole moment intensity causes an upward shift of the Pedersen conductivity profile, this leads to a larger conductance as well as stronger winds (see also Takeda 1996), which should lead to a stronger inverse scaling relation than suggested above. In addition, the neutral wind structure itself has been shown to change with dipole moment intensity (Cnossen et al. 2011) and the electrostatic electric field $\mathbf{E}$ also depends on the dipole moment intensity, strengthening with increasing magnetic field strength (Takeda 1996). Simply scaling the Sq variation as the product of the conductance and the dipole moment intensity is therefore an oversimplification. 
Instead we will rely on results obtained from CMIT simulations carried out by Cnossen et al. (2012a), which take the effects discussed above into account. These indicate that the daily amplitude of the $\mathrm{Sq}$ magnetic variation at $30^{\circ}$ magnetic latitude scales with $M^{-0.85}$ to $M^{-1.06}$ (depending on the component) under moderate solar activity conditions, as shown in Fig. 2. Note that the fits exclude the points for the smallest dipole moment intensity $\left(M=2 \times 10^{22} \mathrm{Am}^{2}\right)$, because the magnetic perturbations, and indeed the ionospherethermosphere system as a whole, start to behave differently for such a weak main field strength. At $30^{\circ}$ magnetic latitude, the northward component is most strongly dependent on the dipole moment intensity, but its dependence on $M$ gets weaker for $25^{\circ}$ magnetic latitude and stronger for $35^{\circ}$ magnetic latitude. The dependencies of the eastward and downward components on $M$ are much less sensitive to magnetic latitude.

Close to the magnetic equator, the nearly horizontal magnetic field there, together with a large difference in Hall and Pedersen conductivity in the lower ionosphere, results in a strongly enhanced eastward current, known as the equatorial electrojet. The strength of the equatorial electrojet is dependent on the so-called Cowling conductivity, $\sigma_{C}$, given by (e.g., Richmond 1995):

$$
\sigma_{C}=\sigma_{P}+\frac{\int \sigma_{H} d s}{\int \sigma_{P} d s} \sigma_{H}
$$

where the integrals are taken along a magnetic fieldline. In the equatorial region, the fieldlines only reach the lower part of the ionosphere, where the collision frequencies are larger than the gyro-frequencies. In this height range, the fieldline-integrated Pedersen conductivity should not depend much on the main field strength at all, while the dependence of the fieldline-integrated Hall conductivity should scale close to $M^{-1}$ (see Eqs. (1a), (1b), (2a), (2b); also Cnossen et al. 2012a). This means that the scaling for the Cowling conductance should be somewhere in between $M^{0}$ and $M^{-2}$, but probably closer to $M^{-2}$ than $M^{0}$, as the Hall conductivity dominates in the lower ionosphere. This is indirectly confirmed by the modelling results of Cnossen et al. (2012a). These indicate that the amplitude of the horizontal component of the daily magnetic variation simulated at the magnetic equator, which we will call $\Delta B_{E E J}$, scales as:

$$
\Delta B_{E E J} \propto M^{-0.7}
$$

for $M$ between $6 \times 10^{22}$ and $10 \times 10^{22} \mathrm{Am}^{2}$. If we assume that this amplitude scales as the product of the Cowling conductance and the main field strength, this implies that the Cowling conductance scales approximately as $M^{-1.7}$. This is in excellent agreement with a scaling for the Cowling conductance previously suggested by Glassmeier et al. (2004).

\subsection{Polar Electrojets}

The ground magnetic perturbations associated with the polar electrojets are mainly determined by the ionospheric Hall currents, because the ground signature of the horizontal Pedersen currents is cancelled by the signature of the (vertical) field-aligned currents, assuming that the ionospheric conductivity is spatially uniform (Fukushima 1976). Although this assumption is not strictly valid in practice, we can use this to derive a first-order scaling relation for the magnetic perturbation associated with the polar electrojet, $\triangle B_{P E J}$, following Glassmeier et al. (2004). They proposed that $\Delta B_{P E J} \propto \Sigma_{H} E_{c}$, where $E_{c}$ is the high-latitude electric field driven by magnetospheric convection. Glassmeier et al. (2004) used further 
assumptions and theoretical arguments to propose a scaling for $E_{c}$ with the dipole moment intensity, arriving at:

$$
E_{c} \propto M^{10 / 3-4 \gamma}
$$

For $\gamma=\frac{1}{3}$ (moderately southward solar wind conditions) this gives $E_{c} \propto M^{2}$. It is also possible to obtain a scaling relation for $E_{c}$ from the CMIT model simulations by Cnossen et al. (2012a), which used IMF $B_{z}=-5 \mathrm{nT}$. To do this, we assume that $E_{c}$ can be approximated by $\Phi / \cos \left(\lambda_{p c}\right)$, where $\Phi$ is the cross-polar cap potential and $\lambda_{p c}$ is the latitude of the polar cap boundary, defined as the boundary between open and closed magnetic field lines $\left(\cos \left(\lambda_{p c}\right)\right.$ is used here as a characteristic length scale for the polar cap). The simulations by Cnossen et al. (2012a) indicate that the quantity $\Phi / \cos \left(\lambda_{p c}\right)$ scales almost linearly with the dipole moment intensity for values of $M$ between $6 \times 10^{22}$ and $10 \times 10^{22} \mathrm{Am}^{2}$. However, it can be approximated closely by the following power law scaling (again valid for $M$ between $6 \times 10^{22}$ and $10 \times 10^{22} \mathrm{Am}^{2}$ ), which has the advantage that it is independent of units:

$$
E_{c} \propto M^{0.6}
$$

Zieger et al. (2006a, 2006b) also examined the dependence of the cross-polar cap potential and polar cap size on the dipole moment intensity, using simulations with the Block Adaptive Tree Solar-wind Roe Upwind Scheme (BATS-R-US) MHD code (Powell et al. 1999) and the analytical Hill model (Hill et al. 1976). For the same IMF $B_{z}(-5 \mathrm{nT})$ they found similar dependencies of $\cos \left(\lambda_{p c}\right)$ and $\Phi$ to Cnossen et al. (2012a). Both modelling studies thus indicate that the theoretically obtained scaling by Glassmeier et al. (2004) for $E_{c}$ (Eq. (11)) is much too strong. Here we will use Eq. (12) as the most suitable scaling for $E_{c}$. Combined with the night-side Hall conductance scaling for the auroral zone given in Eq. (6b), this gives:

$$
\Delta B_{P E J} \propto M^{3(\gamma-1)+0.4}
$$

With $\gamma$ taking values between 0 and $1 / 2$, this indicates that the magnetic signature of the polar electrojet strongly decreases with dipole moment intensity, especially for $\gamma=0$ (strong northward IMF).

In principle, it should also be possible to calculate the magnetic signature of the polar electrojet, and its dependence on the dipole moment intensity, directly from model simulations, such as those carried out by Zieger et al. (2006a, 2006b) and Cnossen et al. (2012a). However, high-latitude magnetic perturbations calculated from more realistic CMIT model simulations carried out by Cnossen and Richmond (2013) have shown rather poor agreement with observed values. We have therefore not analysed the simulated currents at highlatitudes directly, as this could give misleading results. Zieger et al. (2006a, 2006b) did not show results for the magnetic signature of the polar electrojets either.

\subsection{Field-Aligned Currents}

While the contribution of high-latitude field-aligned currents to magnetic perturbations on the ground may be mostly cancelled by horizontal Pedersen currents, they are still of interest for space-based observations and because of their role in coupling the ionosphere and magnetosphere. We therefore briefly discuss them here.

In the first instance, field-aligned currents get stronger for increased conductivity. However, unlike the conductivity, the dependence of the total amount of field-aligned current on the dipole moment intensity does not follow a straightforward power law scaling (e.g., 


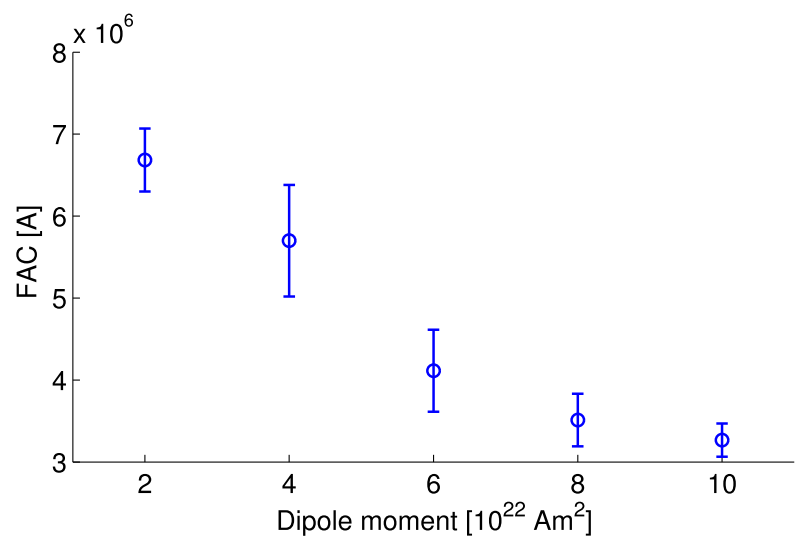

Fig. 3 Dependence of the total field-aligned current (upward + downward), averaged over the two hemispheres, on the dipole moment intensity for intermediate solar activity ( $F 10.7=150 \mathrm{sfu})$ based on CMIT simulations by Cnossen et al. (2012a). Each marker represents a 24-hour average under constant solar wind conditions (Interplanetary Magnetic Field (IMF) $B_{x}=B_{y}=0 ; B_{z}=-5 \mathrm{nT}$; solar wind speed $V_{x}=-400 \mathrm{~km} / \mathrm{s}$; $V_{y}=V_{z}=0$ ), with error bars indicating the standard deviation

Zieger et al. 2006a, 2006b; Cnossen et al. 2012a). This is because a change in dipole moment intensity also affects other aspects of the magnetosphere-ionosphere system, such as the size of the magnetosphere, which additionally influence the field-aligned currents. Figure 3 shows how the total field-aligned current (upward + downward), averaged over the two hemispheres, depends on the dipole moment intensity according to the CMIT simulations by Cnossen et al. (2012a) under moderate solar activity conditions. This represents mainly region- 1 currents, as the model generates only very weak region- 2 currents, due to a poor representation of the ring current.

\subsection{Ring Current}

The surface magnetic field associated with the ring current, $\Delta B_{R C}$, is proportional to the energy of the ring current particles, $E_{r c}$, divided by the dipole moment intensity, $M$ (e.g., Kivelson and Russell 1995):

$$
\Delta B_{R C} \propto \frac{E_{r c}}{M}
$$

Siscoe and Chen (1975) reasoned that the energy of ring current particles can be expected to scale in the same way as the total energy input to the magnetosphere, which should be proportional to the cross-sectional area of the magnetosphere tail, $R_{T}^{2}$. If the magnetosphere retains its shape (i.e., remains self-similar), $R_{T}^{2}$ is proportional to $R_{M P}^{2}$, where $R_{M P}$ is the magnetopause stand-off distance. From a simple pressure balance at the magnetopause between the solar wind dynamic pressure and the magnetic pressure from the Earth's magnetic field, it follows that $R_{m p}$ scales to first order as $M^{1 / 3}$ (e.g., Kivelson and Russell 1995). This then gives:

$$
\Delta B_{R C} \propto R_{T}^{2} / M \propto M^{-1 / 3}
$$

Vogt and Glassmeier (2001) relaxed the assumption of self-similarity, suggesting that the tail radius $R_{T}$ should scale as $M^{\gamma}$. This is where the factor $\gamma$, first introduced in Sect. 2.1, 
comes from. Further, Glassmeier et al. (2004) argued that it is not only the cross-section of the tail of the magnetosphere that matters for the energy of the ring current particles, but also the volume of the part of the magnetosphere where particle trapping is important, $V_{r c}$. Assuming that this volume scales with $M$, they proposed the following scaling for $\Delta B_{R C}$ :

$$
\Delta B_{R C} \propto R_{T}^{2} V_{r c} / M \propto M^{2 \gamma}
$$

Unlike Eq. (14), the Glassmeier et al. (2004) scaling (Eq. (16)) indicates a decrease in $\Delta B_{R C}$ with decreasing dipole moment intensity. This is much more reasonable in the limit of a vanishing main field and is also supported by observations in Mercury's magnetosphere, which indicate that its small magnetic field does not support a large ring current. Having said that, it is still uncertain whether Eq. (16) describes accurately how strongly we can expect $\Delta B_{R C}$ to decrease with decreasing dipole moment intensity. Modelling studies that have been carried out so far to examine effects of changes in dipole moment intensity on the magnetosphereionosphere system (e.g., Zieger et al. 2006a, 2006b; Cnossen et al. 2011, 2012a) did not examine the effects on $\Delta B_{R C}$, because the ring current is not well described by the models they used. Effects of changes in dipole moment intensity on other magnetospheric currents, such as tail and magnetopause currents, have not yet been studied either.

\section{Effects of Changes in Dipole Moment Orientation}

In this section we consider again first the simplified case of a dipolar magnetic field, but this time we discuss effects of the orientation of that dipole. The orientation of the dipole determines the mapping between geographic and magnetic coordinates, and therefore the geographic positioning of important features, such as the magnetic poles, the auroral oval, and the magnetic equator (e.g., Siscoe and Christopher 1975; Cnossen and Richmond 2012). When the dipole orientation changes, these features move in a geographic reference frame, and current systems that are tied to them move along. However, much more than a simple shift of current systems occurs.

First, a change in the geographic location of a current system, in particular the geographic latitude, affects the background conductivity arising from solar extreme ultraviolet (EUV) radiation. Movement to a lower geographic latitude results on average in a higher conductivity, and hence stronger currents. Second, the dynamo electric field, $\mathbf{v} \times \mathbf{B}$, is naturally affected by changes in the direction of the magnetic field, which consequently affects the currents. Further, also the neutral wind can be influenced by a different orientation of the magnetic field, because the ion-drag force on the neutral wind depends on the magnetic field orientation (e.g., Cnossen and Richmond 2008). In addition, the magnetic field orientation affects plasma transport processes, and therefore the plasma distribution and conductivity, additionally influencing the currents.

The effects described above drive asymmetries in conductivities and winds between the Northern and Southern hemisphere when the dipole is not aligned with the Earth's rotation axis, which add to any North-South asymmetries already present due to seasonal variations. Hemispheric asymmetries in conductivities and winds lead to asymmetries in electric potential, which tend to be quickly shorted out by inter-hemispheric currents flowing along the magnetic field at low- to mid-latitudes, due to the high conductivity parallel to the magnetic field. During solstice, inter-hemispheric field-aligned currents flow mainly from the summer to the winter hemisphere at dawn and vice versa around noon (Takeda 1982; Park et al. 2011). The orientation of the magnetic field determines in part how strong these interhemispheric field-aligned currents and the magnetic perturbations associated with them are. 
In addition to the average structure and strength of ionospheric current systems, the orientation of the magnetic field also affects their temporal variations. When the dipole is not aligned with the rotation axis, its daily precession about the rotational axis creates a UT dependence in the ionosphere. The larger the dipole tilt, by which we mean here the angle between the magnetic dipole axis and the Earth's rotation axis, the larger this daily variation becomes. Seasonal variations are similarly modified by the orientation of the dipole axis. Further discussions of these effects are given by, e.g., Walton and Bowhill (1979), Wagner et al. (1980), Richmond and Roble (1987), and more recently Cnossen and Richmond (2012).

Hurtaud et al. (2007) examined how high-latitude region-2 field-aligned currents depend on the dipole orientation, studying three different cases: a) a dipole aligned with the Earth's rotation axis, b) a dipole tilted with respect to the rotation axis, and c) a dipole that is additionally offset from the centre of the Earth. Simulations with the Ionosphere Magnetosphere Model (Peymirat and Fontaine 1994) showed that the introduction of a dipole tilt causes stronger diurnal and seasonal variations in region-2 field-aligned currents, due to increased diurnal and seasonal variations in ionospheric conductivity, as expected. The offset from the centre of the Earth reduced the variations in one hemisphere (where the magnetic pole was closer to the geographic pole), while enhancing them in the other. This effect is also described by Laundal et al. (2016) in the context of North-South hemispheric differences.

Currents in the wider magnetosphere may also show some dependence on dipole orientation, because this determines in part the geometry between the Earth's magnetic field and the solar wind and the IMF, which can affect the solar wind-magnetosphere coupling efficiency (e.g., Cnossen et al. 2012b). However, little is known about the effects of this on magnetospheric current systems. In principle, naturally occurring diurnal and seasonal variations in the orientation of the main dipole component of the Earth's present-day magnetic field with respect to the solar wind and IMF could provide some information, but in practice these effects tend to be swamped by the large variability in solar wind IMF conditions. A modelling study by Zieger et al. (2004) of a rather extreme change in dipole orientation does offer some insights. They adapted the BATS-R-US model to simulate the magnetosphere under the condition of a dipole that is oriented perpendicular to the Earth's rotation axis. This resulted in dramatic daily variations in the magnetopause currents and tail current sheet. However, they stressed that bowshock and magnetosheath currents remained more or less the same, as these are controlled by the IMF orientation.

When we move away from a simple dipolar magnetic field structure, it becomes more complicated to describe what happens to current systems in the ionosphere and magnetosphere. For the magnetosphere, the non-dipolar contributions to the magnetic field are less important as these contributions diminish quickly with distance from the Earth. However, in the ionosphere there are notable regional variations that are associated with non-dipolar structures of the magnetic field. Some of the effects of non-dipole components on lowlatitude currents were discussed by Walton and Bowhill (1979), while Siscoe and Sibeck (1980) described effects on the auroral zone. Gasda and Richmond (1998) discussed the influence of non-dipolar structures on auroral electrodynamics in further detail, including implications for the auroral electrojets and field-aligned currents. They predicted considerable longitudinal variations in electrojet current densities, with differences between local maxima and minima of about $40 \%$ in the Northern hemisphere. Le Sager and Huang (2002) used model simulations to study how the Sq current system changes when the full IGRF magnetic field structure is used to define the main field, as opposed to an aligned or tilted dipole. A realistic magnetic field resulted in considerably different horizontal and fieldaligned low- to mid-latitude currents, while some features were not simulated at all with the 
dipole approximations. For instance, a time lag of $\sim 1$ hour between the horizontal current foci in the Northern and Southern hemispheres at equinox, which had previously been found in observations, could only be (approximately) reproduced with the full IGRF.

\section{Effects of Realistic Magnetic Field Changes in the Past 100 Years}

In this section we will examine the effects of changes in the actual magnetic field between 1908 and 2008, based on CMIT simulations carried out by Cnossen and Richmond (2013) and similarly set up TIE-GCM simulations by Cnossen (2014). All of these simulations were run with background geophysical conditions of 2008, when solar and geomagnetic activity were generally low. Figure 4 a illustrates how the magnetic field intensity changed between 1908 and 2008, according to the IGRF. The main dipole component decreased in intensity from $\sim 8.3 \times 10^{22} \mathrm{Am}^{2}$ in 1908 to $\sim 7.7 \times 10^{22} \mathrm{Am}^{2}$ in 2008 , i.e., a very small change in comparison to the large range of values explored in Sect. 2. However, the changes in magnetic field strength are far from globally uniform and are still rather large (up to nearly $40 \%$ ) in some regions, such as South America and the southern Atlantic Ocean. This relates to the expansion of the South Atlantic Anomaly region. Figure $4 \mathrm{~b}$ shows the movement of some of the key features of the magnetic field, such as the magnetic dip equator, the magnetic dip poles, and several constant inclination contours in between. Again, the strongest changes have occurred over the Atlantic Ocean.

The effects of these changes in the magnetic field on the ionospheric Pedersen conductance, as simulated by Cnossen (2014) with the TIE-GCM, are illustrated in Fig. 5. Two different UTs are shown. At 0 UT it is night-time in the region where the magnetic field has changed the most, which results in much smaller changes in Pedersen conductance at low to mid-latitudes than at $12 \mathrm{UT}$, when it is day-time in this region. The low- to mid-latitude changes in day-time Pedersen conductivity appear to be caused primarily by changes in the main field strength, as they are in good agreement with the scaling relation of Eq. (5a). At high latitudes, the changes in Pedersen conductance are much less dependent on local time, although they appear slightly stronger at night than during the day between $\sim 90^{\circ} \mathrm{W}$ and $60^{\circ}$ E. Similar spatial patterns of change and local time dependencies can be found for the Hall conductance (not shown).

The Sq current system is affected by changes in the ionospheric conductivities, but also by the change in the geographic positioning of the magnetic equator and other magnetic structures. This effect is clearly seen in the equivalent current function, shown in Fig. 6 (shown for 12 UT only). The contours of the equivalent current function correspond to flow lines of the equivalent current system. Changes in the Sq currents further lead to changes in the associated ground magnetic perturbations, shown in Fig. 7 (for 0 and 12 UT). Because the Sq system is a daytime phenomenon, changes are again more prominent around $12 \mathrm{UT}$ than around 0 UT. Over South America, the Atlantic Ocean, and Africa, changes are particularly large: magnetic perturbations in all three directions are easily a factor 2 different between 1908 and 2008, and locally this can be even more.

Figure 8 further illustrates the effects of changes in the main magnetic field on the daily magnetic variation at three example locations, which experienced different types of main magnetic field changes (see Fig. 4). At Apia $\left(13.8^{\circ} \mathrm{S}, 171.8^{\circ} \mathrm{W}\right)$ there have only been small changes in the main field strength $(\sim 5 \%$ decrease $)$ and field orientation $\left(\sim 2^{\circ}\right.$ in inclination and declination), which produced no significant change in the daily Sq variation. At Huancayo $\left(12.0^{\circ} \mathrm{S}, 75.3^{\circ} \mathrm{W}\right)$ there has been a much larger decrease in the main field strength 

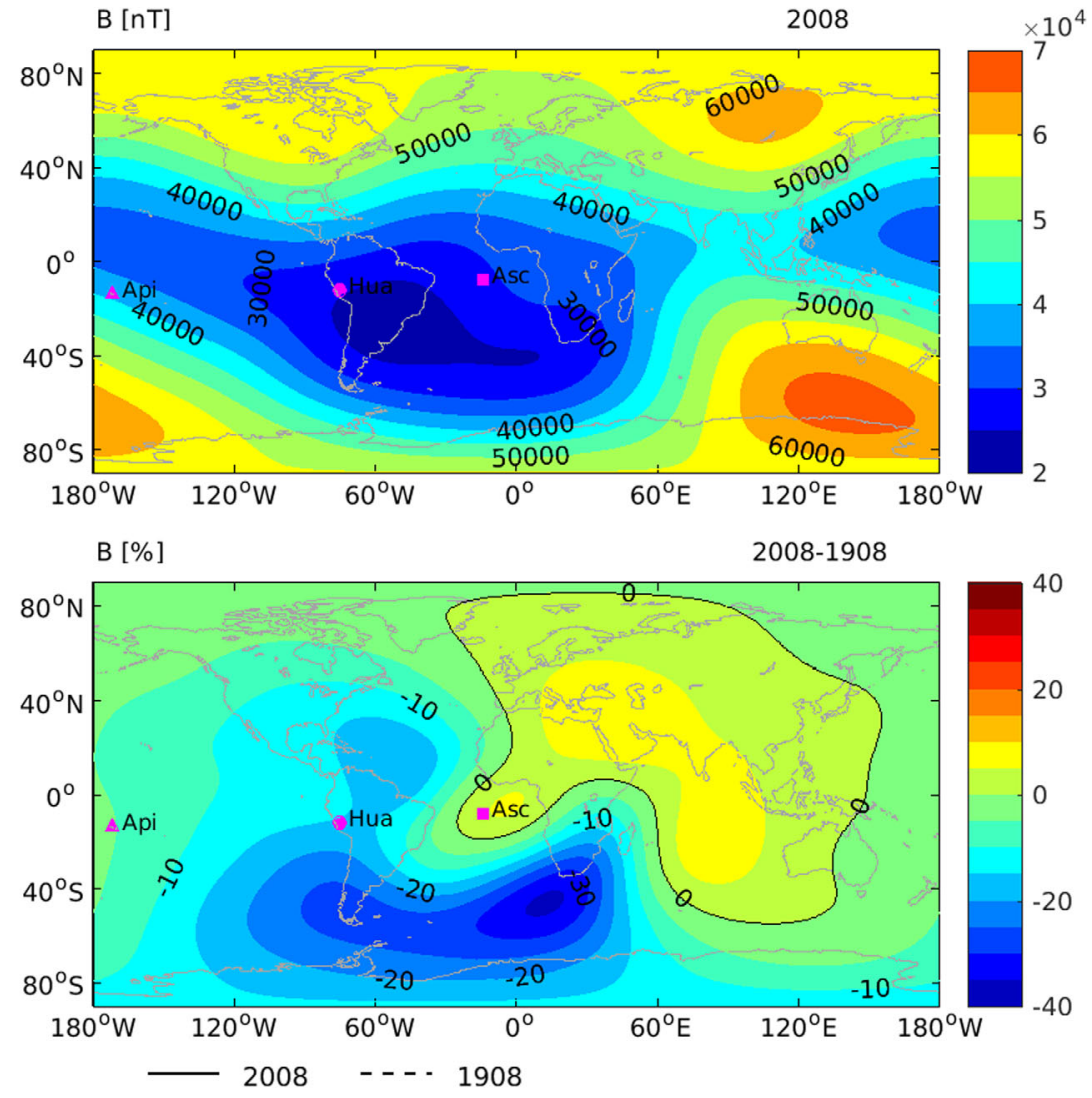

(a)

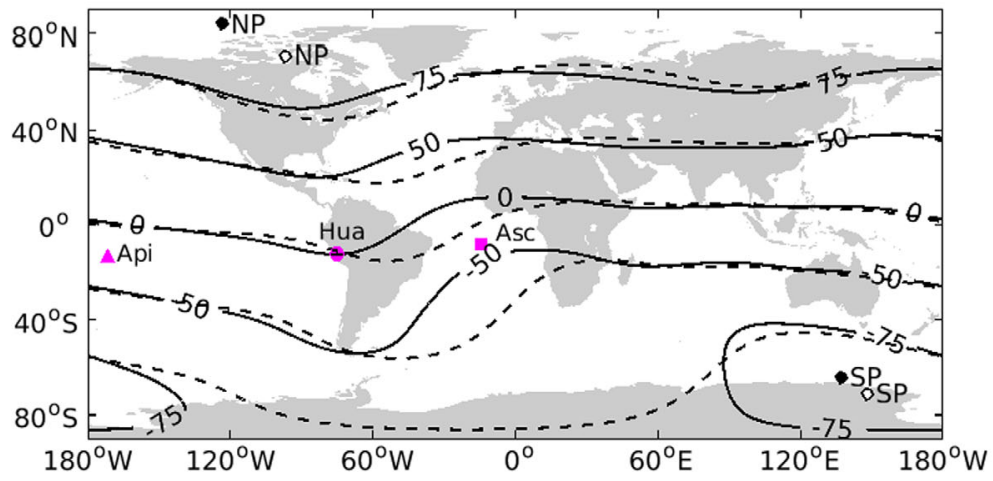

(b)

Fig. 4 (a) Main magnetic field intensity (nT) in 2008 (top) and the percentage difference between 2008 and 1908 (bottom) according to the IGRF. Magenta markers indicate the locations of Apia (triangle), Huancayo (circle) and Ascension Island (square), which will be discussed later in the text. (b) Contours of constant magnetic field inclination $\left({ }^{\circ}\right)$ for 2008 (solid lines) and 1908 (dashed lines). The positions of the magnetic dip poles are indicated by solid black (2008) and open (1908) circles. Magenta markers indicate the locations of Apia (triangle), Huancayo (circle) and Ascension Island (square), which will be discussed later in the text 
Fig. 5 (a) Average Pedersen conductance (S) for 2008 (top) and the difference with 1908 (2008-1908; bottom) at 0 UT for equinox conditions, based on TIE-GCM simulations by Cnossen (2014). Light (dark) shading indicates where differences are statistically significant at the $95 \%(99 \%)$ level according to a two-sided $t$-test. (b) Same as (a), but for 12 UT
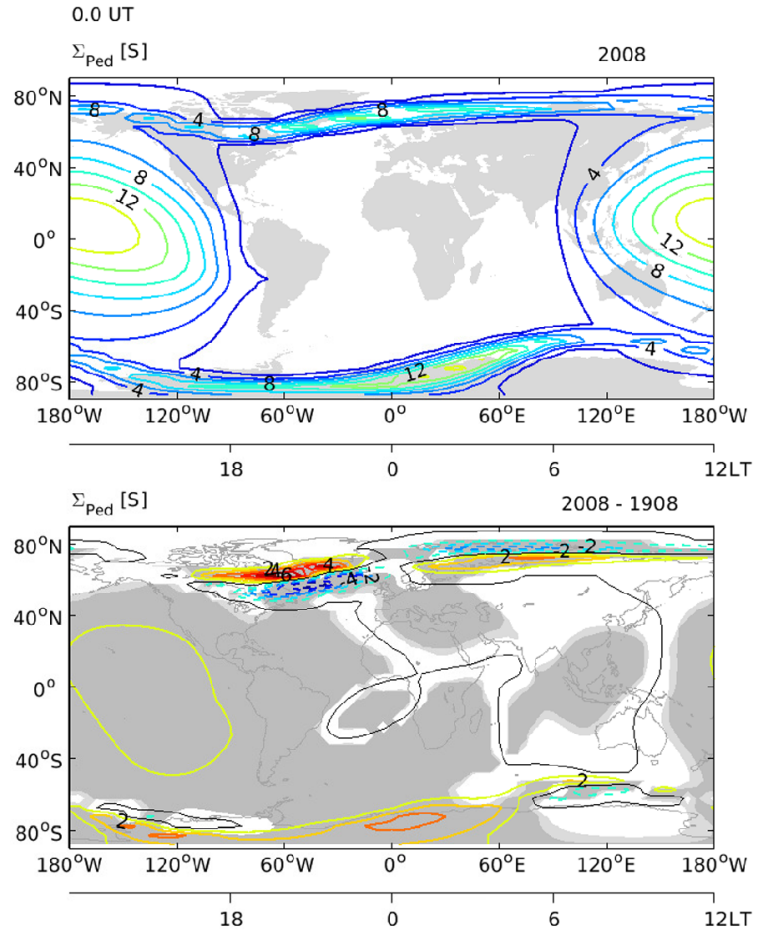

(a)
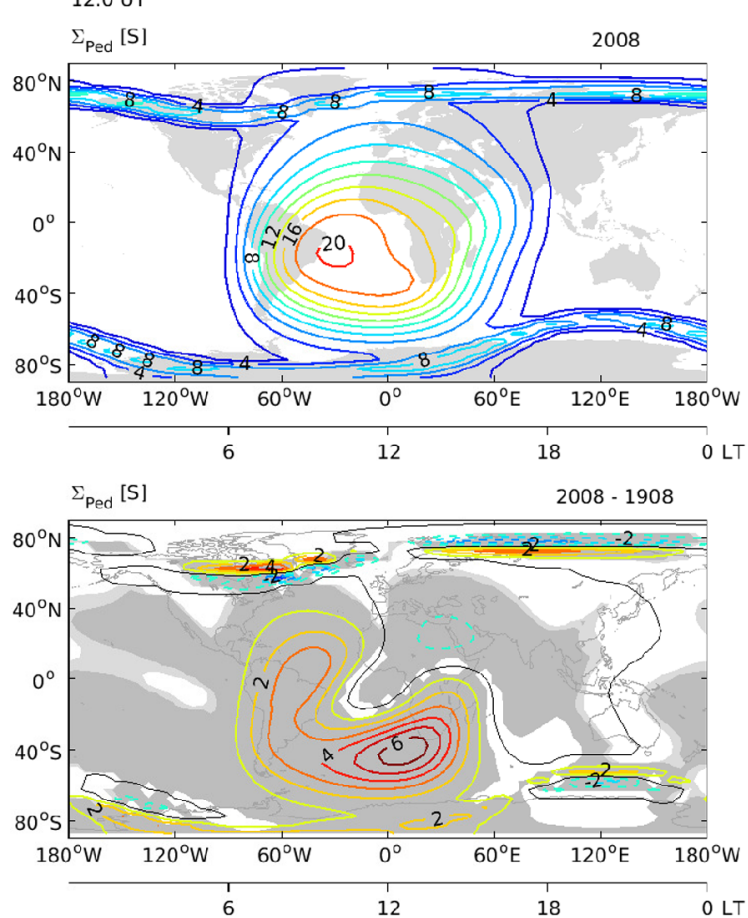

(b) 
Fig. 6 Average equivalent current function (kA) for 2008 (solid lines) and 1908 (dashed lines) at 12 UT for equinox conditions, based on TIE-GCM simulations by Cnossen (2014)

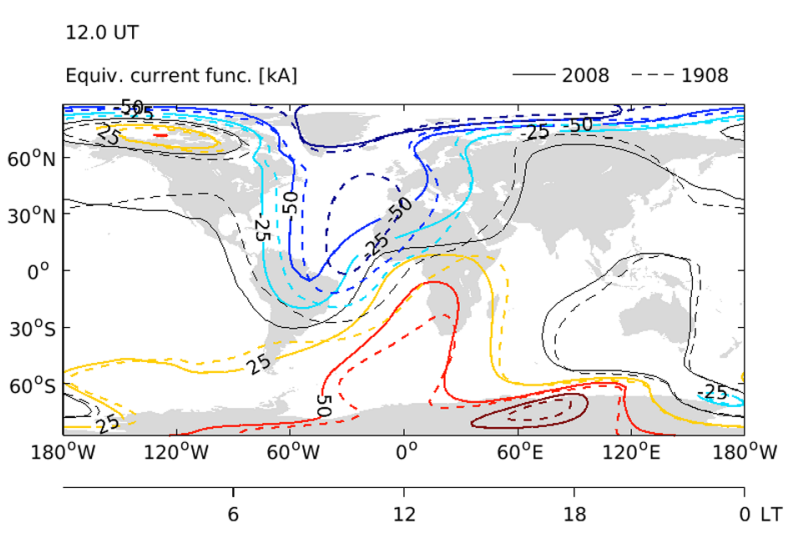

(15-20\%), combined with little change in inclination. The changes in Sq variation at Huancayo shown in Fig. 8 can therefore be attributed mainly to the decrease in the main field strength, although the local time shift in the daily variation of the eastward component is probably associated with a substantial change in declination $\left(\sim 10^{\circ}\right)$. In contrast, at Ascension Island $\left(7.9^{\circ} \mathrm{S}, 14.4^{\circ} \mathrm{W}\right)$ the decrease in the main field strength is small $(\sim 5 \%)$, while the inclination angle has changed by more than a factor 2 as the magnetic equator has moved away from this location since 1908. This movement of the magnetic equator is primarily responsible for the changes in Sq variation we find at Ascension Island. Observational studies have also found evidence for long-term trends in Sq variation, which to some degree, depending on location, have been attributed to main magnetic field changes (Elias et al. 2010; De Haro Barbas et al. 2013).

We note that Fig. 7 also shows fairly large changes in magnetic perturbations at high latitudes, which appear significant. However, the TIE-GCM does not fully represent the high-latitude current systems that contribute to magnetic perturbations in these regions, so that this result is much less reliable than the results for low- and mid-latitudes. The CMIT model should be able to capture some of the high-latitude current systems somewhat better than the TIE-GCM can, because it includes a representation of the magnetosphere, but as noted before, the high-latitude currents are still challenging to simulate correctly.

CMIT simulations similar to the TIE-GCM simulations discussed above indicate that there is no significant difference in the total field-aligned current between 1908 and 2008 for either the Northern or Southern hemisphere. In terms of spatial structure, there are no significant differences either when viewed in magnetic coordinates, but when transformed to geographic coordinates, differences do appear due to the movement of the magnetic poles. This is illustrated in Fig. 9 for 0 UT. Especially in the Northern hemisphere there has been a clear shift in the average field-aligned current pattern from 1908 to 2008. Similar shifts are seen for other UTs.

\section{Implications for Internal Magnetic Field Modelling}

The previous sections have shown that the secular variation of the internal magnetic field causes changes in the currents flowing in the magnetosphere and ionosphere, thereby inducing a long-term change in the externally produced magnetic field. This poses a problem for internal magnetic field modellers who seek to model the secular variation of the internal magnetic field, as the observed magnetic field contains contributions from both internal 

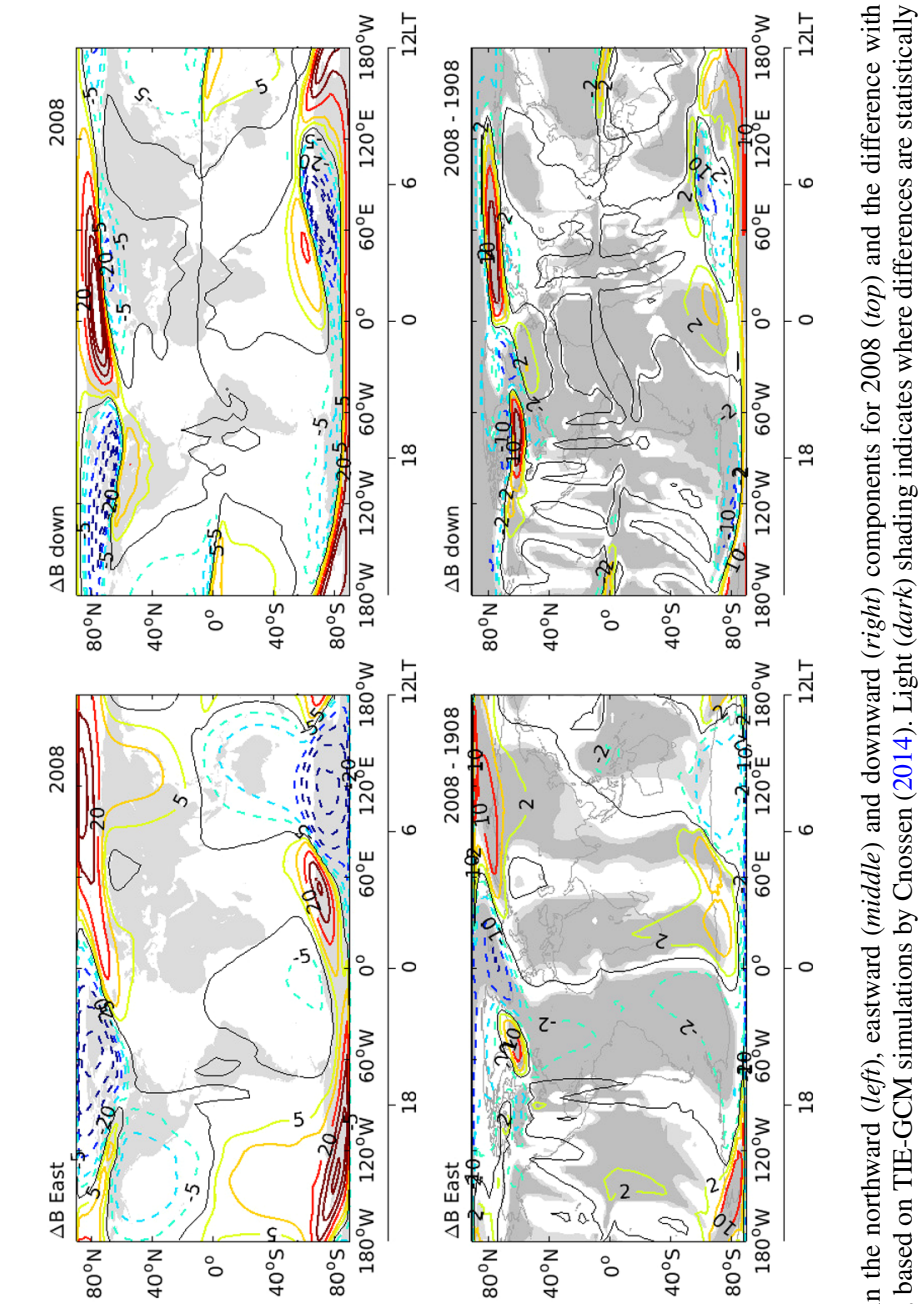

$=$

हี

웡 류 ญิ 츤

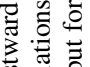
芯菉 $\therefore$ 需

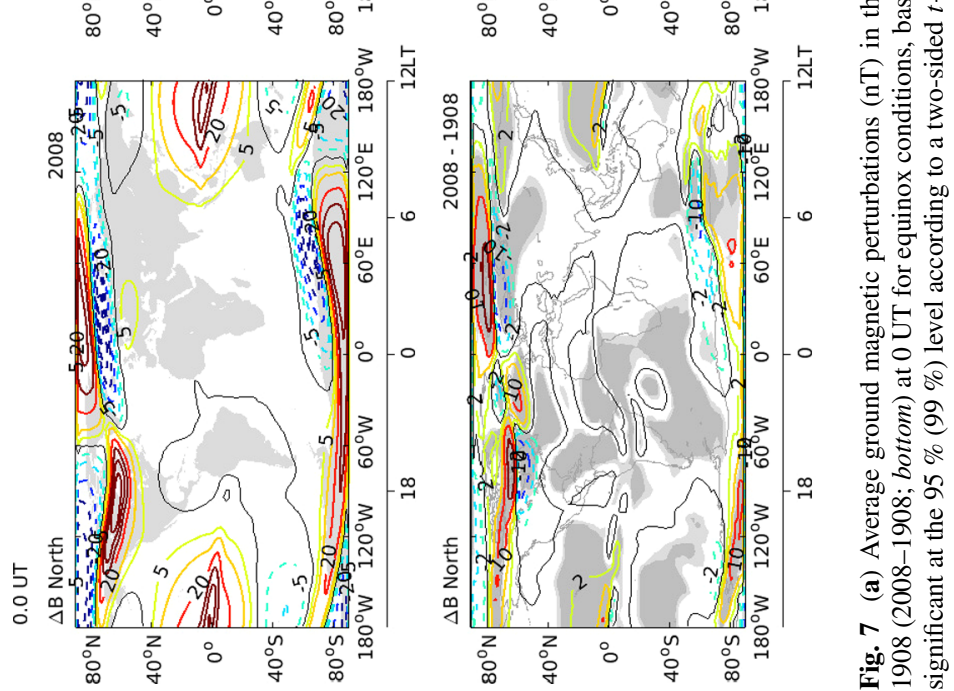

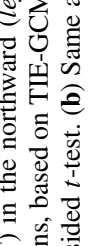

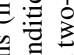
ర 兽.” 苍 可与 ส कू ฐ țํㅇ 光 융 용 苛 

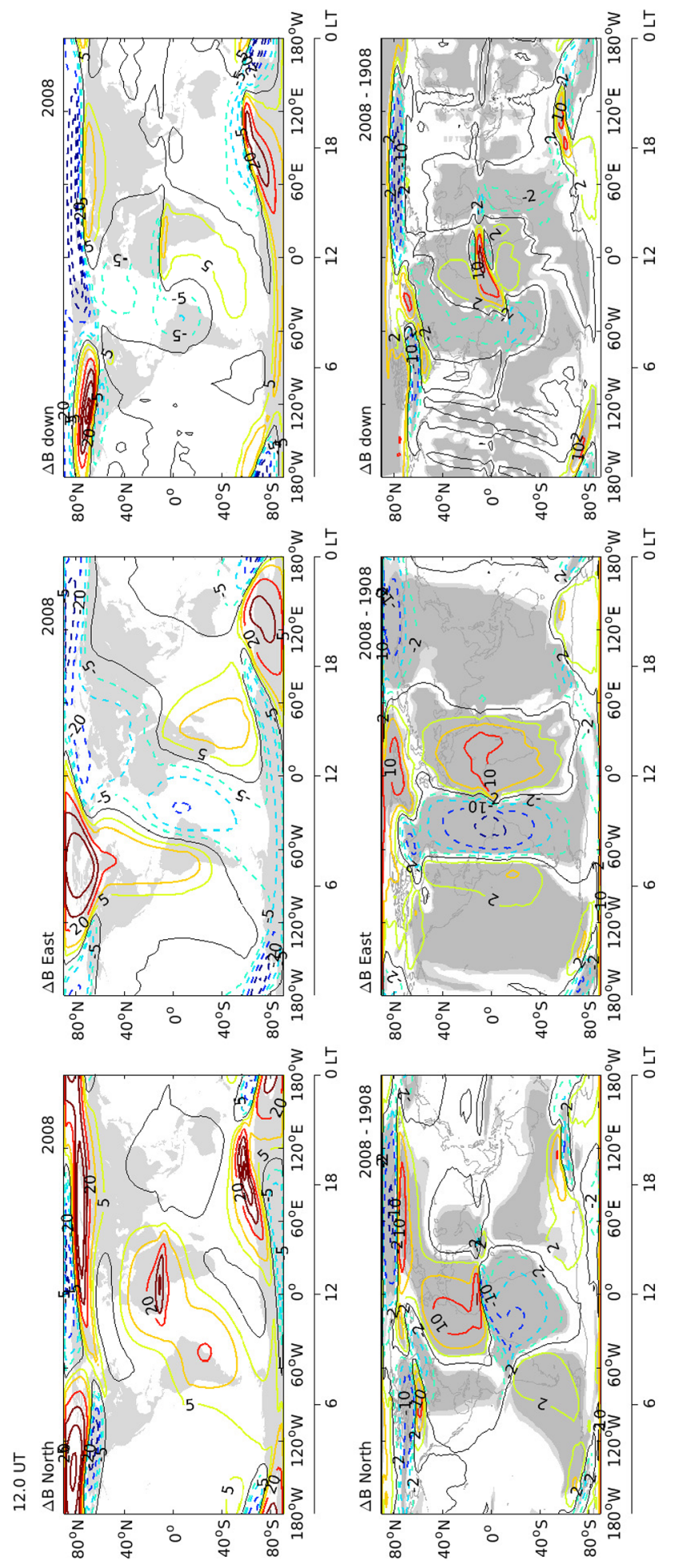

 
Fig. 8 Average daily magnetic perturbations in the northward (top), eastward (middle) and downward (bottom) components for 2008 (solid lines) and 1908 (dashed lines) at Huancayo (black), Apia (red), and Ascension Island (blue), based on TIE-GCM simulations by Cnossen (2014). Shading in corresponding colour tones indicates the $95 \%$ confidence interval
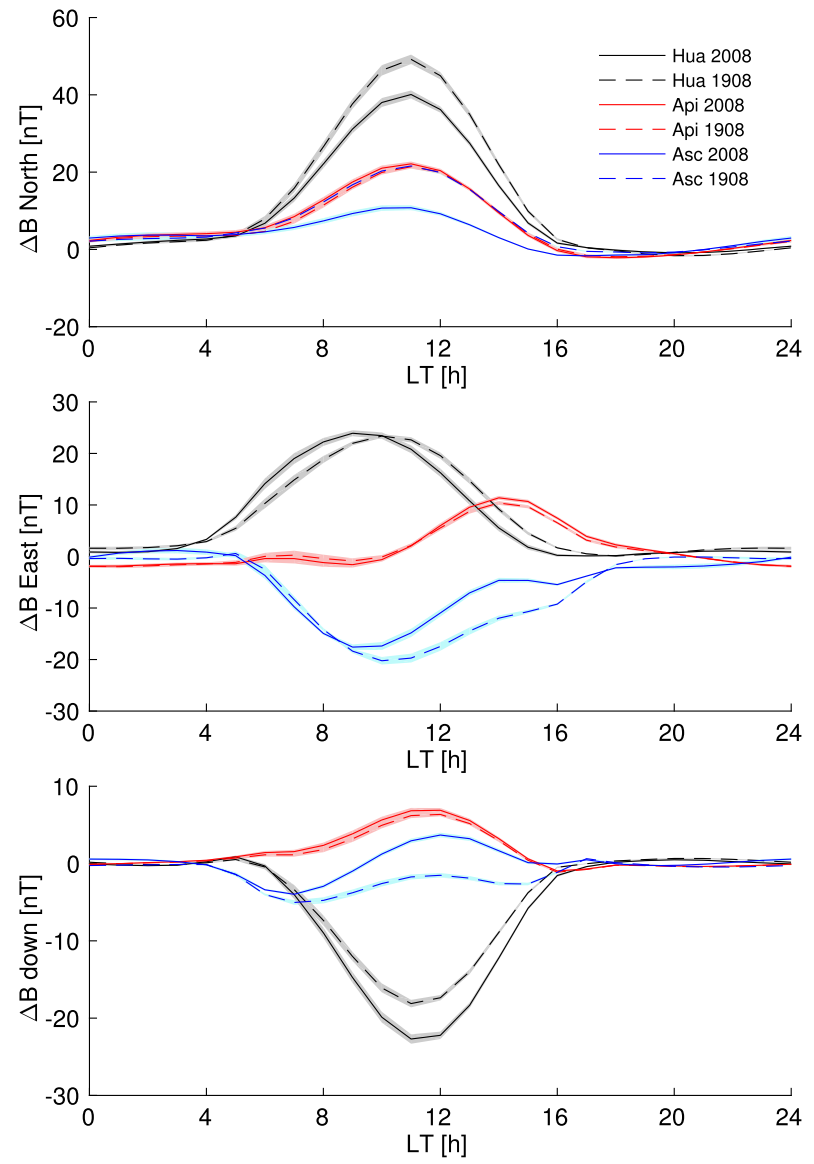

and external sources. How long-term changes in ionospheric and magnetospheric current systems affect the modelling of the secular variation has not been studied before. However, the previous sections indicate that the degree to which long-term changes in the externally induced magnetic field can leak into models of the secular variation of the internal magnetic field depends on the current system in question as well as the approach taken to model the internal magnetic field, in particular the data selection criteria used. Some strategies to minimize effects of long-term changes in external currents associated with main magnetic field changes in internal magnetic field modelling are discussed below.

The Sq current system is primarily important during the day-time, and therefore the impact of any long-term changes in the magnetic perturbations associated with this current system will be small when only night-time data are used to construct an internal magnetic field model. This is the approach taken for the majority of internal magnetic field models (see Finlay et al., this volume, 2016). However, if day-time data are included, as is done, for example, in the Comprehensive Model (CM) series (Sabaka et al. 2002, 2004), longterm changes in the Sq current system will be relevant. Fortunately, it should be relatively straightforward to account for this effect as part of the model. The CM series includes a model of the Sq current system in quasi-dipole coordinates, so that the influence of any changes in the positioning of the magnetic equator will automatically be taken into account. The influence of any long-term change in the main field strength is currently not included, 


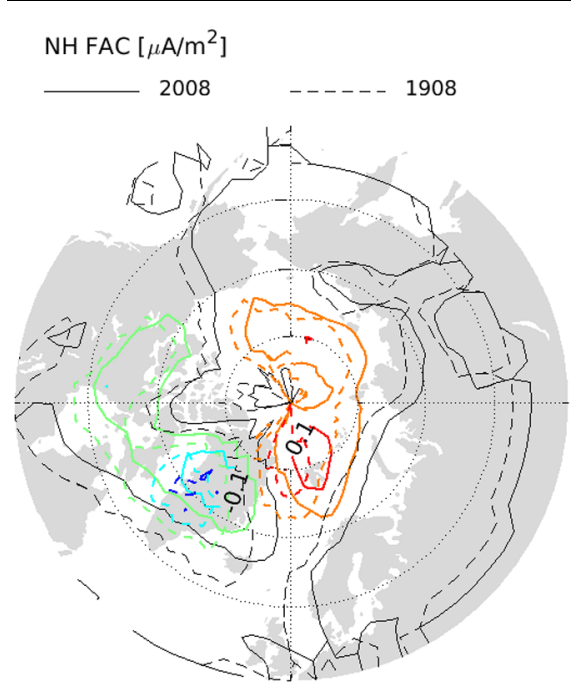

0.0 UT
$\mathrm{SH}$ FAC $\left[\mu \mathrm{A} / \mathrm{m}^{2}\right]$

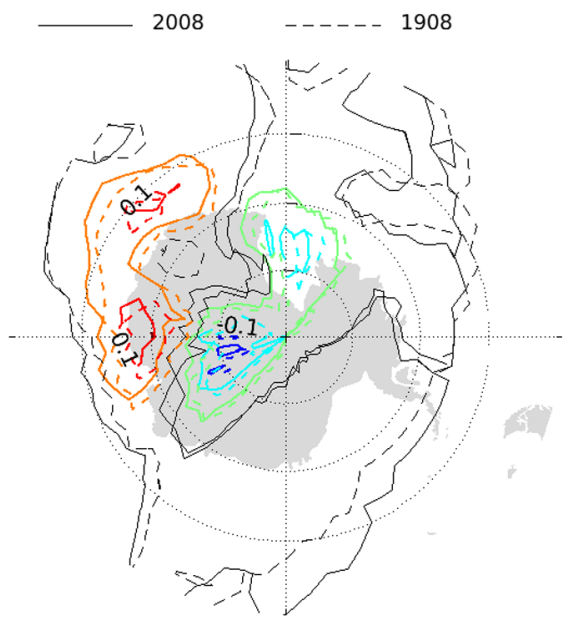

0.0 UT

Fig. 9 Average field-aligned current patterns $\left(\mu \mathrm{A} / \mathrm{m}^{2}\right)$ in the Northern hemisphere (left) and Southern hemisphere (right) for 2008 (solid lines) and 1908 (dashed lines) at 0 UT, based on CMIT simulations as set up by Cnossen and Richmond (2013), but extended to a total of 28 days surrounding March equinox. Contour level spacing is $0.05 \mu \mathrm{A} / \mathrm{m}^{2}$

but could be considered using scaling relations between the magnetic signatures of the Sq current system and the main field intensity as shown in Fig. 2.

The sensitivity of the magnetic perturbations associated with the ring current and the polar electrojets to main magnetic field changes is dependent not only on whether it is dayor night-time, but also on the solar wind conditions, as expressed through the factor $\gamma$ in the scaling relations derived in Sect. 2. Since these current systems also depend on solar wind conditions on short timescales, some internal magnetic field models already employ data selection criteria based on the $B_{y}$ and $B_{z}$ component of the IMF to minimize their influence (e.g., Finlay et al., this volume, 2016). When only data for northward IMF are used, Eq. (16) (with $\gamma=0$ ) indicates that the contribution of the ring current should not be sensitive to any change in the main dipole moment intensity, so this appears to be an effective way to minimize contamination of both the instantaneous internal magnetic field and its secular variation by the ring current contribution. In contrast, Eq. (13) suggests that the magnetic signature of the polar electrojet is strongly dependent on the main dipole moment intensity for northward IMF. With $\gamma=0$ we obtain $\Delta B_{P E J} \propto M^{-2.6}$, which gives a $\sim 30 \%$ increase in the polar electrojet signature for a $10 \%$ decrease in the main field strength. Given that magnetic perturbations associated with the polar electrojets are already very hard to model and separate from the internal magnetic field (Finlay et al., this volume, 2016) avoiding leakage of this external magnetic field contribution into the secular variation of the main field will be extremely challenging.

\section{Summary and Conclusions}

We have obtained scaling relations to describe how various external magnetic field contributions depend on the internal magnetic field strength, based on theoretical arguments in 
combination with modelling studies. Scaling relations for the daytime Pedersen and Hall conductances (see Eqs. (5a), (5b)) can be considered fairly reliable, but night-time scalings in the auroral zone (Eqs. (6a), (6b)) are more uncertain. This is because the night-time conductances in the auroral zone depend strongly on energetic particle precipitation from the magnetosphere, and it is still not well understood how the flux and energy of these particles might change under the influence of a changing main magnetic field. This problem also feeds into the scaling for the magnetic perturbations associated with the polar electrojets (Eq. (13)) and estimates of changes in field-aligned currents with a changing dipole moment intensity (Fig. 3).

Changes in the orientation of a dipolar magnetic field affect the mapping between magnetic and geographic coordinates, and therefore the interaction between processes that are mainly organized in a magnetic reference frame (e.g., magnetosphere-ionosphere coupling, ion drag) and processes that are mainly organized in a geographic reference frame (e.g., solar EUV-driven ionization and heating). When the geographic and dipole axes are not aligned, this further introduces longitudinal and UT variations in the ionosphere and produces asymmetries between the Northern and Southern hemisphere which lead to interhemispheric currents flowing at low- to mid-latitudes.

Departures from a simple dipolar structure cause more complex changes to ionospheric currents. This is illustrated by the simulated changes in the Sq current system due to main magnetic field changes between 1908 and 2008. Locally large decreases in main field strength over South America and the southern Atlantic Ocean, as well as the shift of the magnetic equator in the Atlantic sector, have caused substantial changes in the daily magnetic variation, in particular during daytime, while in other parts of the world there is little difference between 1908 and 2008. At night the simulated differences in magnetic perturbations are generally quite small, also in regions where the magnetic field has changed considerably, although they may still be statistically significant.

Further work is needed to improve model estimates of magnetic perturbations at highlatitudes, in particular at night-time. This is not only important for the long-term variations we have focused on here, but also to account for external magnetic field variations on shorter timescales. Improvements to the treatment of energetic particle precipitation (e.g., Zhang et al. 2015) in models will likely help with this. Enhanced resolution and coupling a model such as the LFM or CMIT to a model of the ring current, can also substantially improve the representation of field-aligned currents (e.g., Korth et al. 2004; Wiltberger et al. 2016). Future studies should test whether with such improvements it is possible to obtain better agreement between observed and simulated magnetic perturbations at high latitudes.

Acknowledgements Part of this work was sponsored by a fellowship of the Natural Environment Research Council, grant number NE/J018058/1. I am grateful to Arthur D. Richmond for helpful comments on an earlier draft of the manuscript and to Christopher C. Finlay for useful discussions on the content of Sect. 5.

\section{References}

I. Cnossen, The importance of geomagnetic field changes versus rising $\mathrm{CO}_{2}$ levels for long-term change in the upper atmosphere. J. Space Weather Space Clim. 4, A18 (2014)

I. Cnossen, A.D. Richmond, Modelling the effects of changes in the Earth's magnetic field from 1957 to 1997 on the ionospheric hmF2 and foF2 parameters. J. Atmos. Sol.-Terr. Phys. 70, 1512-1524 (2008)

I. Cnossen, A.D. Richmond, How changes in the tilt angle of the geomagnetic dipole affect the coupled magnetosphere-ionosphere-thermosphere system. J. Geophys. Res. 117, A10317 (2012)

I. Cnossen, A.D. Richmond, Changes in the Earth's magnetic field over the past century: effects on the ionosphere-thermosphere system and solar quiet (Sq) magnetic variation. J. Geophys. Res. 118, 849858 (2013) 
I. Cnossen, A.D. Richmond, M. Wiltberger, W. Wang, P. Schmitt, The response of the coupled magnetosphere-ionosphere-thermosphere system to a $25 \%$ reduction in the dipole moment of the Earth's magnetic field. J. Geophys. Res. 116, A12304 (2011). doi:10.1029/2011JA017063

I. Cnossen, A.D. Richmond, M. Wiltberger, The dependence of the coupled magnetosphere-ionospherethermosphere system on the Earth's magnetic dipole moment. J. Geophys. Res. 117, A05302 (2012a). doi: $10.1029 / 2012$ JA017555

I. Cnossen, M. Wiltberger, J.E. Ouellette, The effects of seasonal and diurnal variations in the Earth's magnetic dipole orientation on solar wind-magnetosphere-ionosphere coupling. J. Geophys. Res. 117, A11211 (2012b)

B.F. De Haro Barbas, A.G. Elias, I. Cnossen, M. Zossi de Artigas, Long-term changes in solar quiet (Sq) geomagnetic variations related to Earth's magnetic field secular variation. J. Geophys. Res. Space Phys. 118, 3712-3718 (2013)

A.G. Elias, M. Zossi de Artigas, B.F. De Haro Barbas, Trends in the solar quiet geomagnetic field variation linked to the Earth's magnetic field secular variation and increasing concentrations of greenhouse gases. J. Geophys. Res. 115, A08316 (2010)

C.C. Finlay, V. Lesur, E. Thébault, F. Vervelidou, A. Morschhauser, R. Shore, Challenges handling magnetospheric and ionospheric signals in internal geomagnetic field modelling. Space Sci. Rev., in review (this volume, 2016)

N. Fukushima, Generalized theorem of no ground magnetic effect of vertical current connected with Pedersen currents in the uniform conductivity ionosphere. Rep. Ionos. Space Res. Jpn. 30, 35-40 (1976)

S. Gasda, A.D. Richmond, Longitudinal and interhemispheric variations of auroral ionospheric electrodynamics in a realistic geomagnetic field. J. Geophys. Res. 103, 4011-4021 (1998)

K.-H. Glassmeier, J. Vogt, A. Stadelmann, S. Buchert, Concerning long-term geomagnetic variations and space climatology. Ann. Geophys. 22(10), 3669-3677 (2004)

T.W. Hill, A.J. Dessler, R.A. Wolf, Mercury and Mars: the role of ionospheric conductivity in the acceleration of magnetospheric particles. Geophys. Res. Lett. 3, 429-432 (1976)

Y. Hurtaud, C. Peymirat, A.D. Richmond, Modeling seasonal and diurnal effects on ionospheric conductances, region- 2 currents, and plasma convection in the inner magnetosphere. J. Geophys. Res. 112, A09217 (2007)

M.G. Kivelson, C.T. Russell, Introduction to Space Physics (Cambridge University Press, Cambridge, 1995). $568 \mathrm{pp}$.

H. Korth, B.J. Anderson, M.J. Wiltberger, J.G. Lyon, P.C. Anderson, Intercomparison of ionospheric electrodynamics from the iridium constellation with global MHD simulations. J. Geophys. Res. 109, A07307 (2004)

K.M. Laundal, I. Cnossen, S.E. Milan, S.E. Haaland, J. Coxon, N.M. Pedatella, M. Förster, J.P. Reistad, North-South asymmetries: effects on high-latitude geospace. Space Sci. Rev. (2016). doi:10.1007/ s11214-016-0273-0

P. Le Sager, T.S. Huang, Ionospheric currents and field-aligned currents generated by dynamo action in an asymmetric magnetic field. J. Geophys. Res. 107, 1025 (2002)

J.G. Lyon, J.A. Fedder, C.M. Mobarry, The Lyon-Fedder-Mobarry (LFM) global MHD magnetospheric simulation code. J. Atmos. Sol.-Terr. Phys. 66(15-16), 1333-1350 (2004)

J. Park, H. Lühr, K.W. Min, Climatology of the inter-hemispheric field-aligned current system in the equatorial ionosphere as observed by CHAMP. Ann. Geophys. 29, 573-582 (2011)

C. Peymirat, D. Fontaine, Numerical simulation of magnetospheric convection including the effect of fieldaligned currents and electron precipitation. J. Geophys. Res. 99, 11,155-11,176 (1994)

K.G. Powell, P.L. Roe, T.J. Linde, T.I. Gombosi, D.L. De Zeeuw, A solution-adaptive upwind scheme for ideal magnetohydrodynamics. J. Comput. Phys. 154, 284-309 (1999)

A.D. Richmond, Ionospheric electrodynamics, in Handbook of Ionospheric Electrodynamics, Vol. II (1995), pp. 249-290

A.D. Richmond, R.G. Roble, Electrodynamic effects of thermospheric winds from the NCAR thermospheric general circulation model. J. Geophys. Res. 92, 12,365-12,376 (1987)

A.D. Richmond, E.C. Ridley, R.G. Roble, A thermosphere/ionosphere general circulation model with coupled electrodynamics. Geophys. Res. Lett. 19, 601-604 (1992)

H. Rishbeth, The quadrupole ionosphere. Ann. Geophys. 3, 293-298 (1985)

R.G. Roble, E.C. Ridley, A.D. Richmond, A coupled thermosphere/ionosphere general circulation model. Geophys. Res. Lett. 15, 1325-1328 (1988)

T.J. Sabaka, N. Olsen, R.A. Langel, A comprehensive model of the quiet-time, near-Earth magnetic field: phase 3. Geophys. J. Int. 151, 32-68 (2002)

T.J. Sabaka, N. Olsen, M.E. Purucker, Extending comprehensive models of the Earth's magnetic field with ørsted and CHAMP data. Geophys. J. Int. 159, 521-547 (2004)

G.L. Siscoe, C.-K. Chen, The paleomagnetosphere. J. Geophys. Res. 80(14), 4675-4680 (1975) 
G.L. Siscoe, L. Christopher, Effects of geomagnetic dipole variations on the auroral zone locations. J. Geomagn. Geoelectr. 27, 485-489 (1975)

G.L. Siscoe, D.G. Sibeck, Effects of nondipole components on auroral zone configurations during weak dipole field epochs. J. Geophys. Res. 85, 3549-3556 (1980)

G.L. Siscoe, G.M. Erickson, B.U.O. Sonnerup, N.C. Maynard, J.A. Schoendorf, K.D. Siebert, D.R. Weimer, W.W. White, G.R. Wilson, Hill model of transpolar potential saturation: comparisons with MHD simulations. J. Geophys. Res. 107(A6), 1075 (2002)

M. Takeda, Three dimensional ionospheric currents and field aligned currents generated by asymmetrical dynamo action in the ionosphere. J. Atmos. Terr. Phys. 44, 187-193 (1982)

M. Takeda, Effects of the strength of the geomagnetic main field strength on the dynamo action in the ionosphere. J. Geophys. Res. 101, 7875-7880 (1996)

E. Thébault, C.C. Finlay, C. Beggan et al., International geomagnetic reference field: the 12th generation. Earth Planets Space 67, 79 (2015)

J. Vogt, K.-H. Glassmeier, Modelling the plaeomagnetosphere: strategy and first results. Adv. Space Res. 28, 863-868 (2001)

C.-U. Wagner, D. Möhlmann, K. Schäfer, V.M. Mishin, M.I. Matveev, Large-scale electric fields and currents and related geomagnetic variations in the quiet plasmasphere. Space Sci. Rev. 26, 391-446 (1980)

E.K. Walton, S.A. Bowhill, Seasonal variations in the low latitude dynamo currents system near sunspot maximum. J. Atmos. Terr. Phys. 41, 937-949 (1979)

W. Wang, M. Wiltberger, A.G. Burns, S.C. Solomon, T.L. Killeen, N. Maruyama, J.G. Lyon, Initial results from the coupled magnetosphere-ionosphere-thermosphere model: thermosphere-ionosphere responses. J. Atmos. Sol.-Terr. Phys. 66, 1425-1441 (2004)

W. Wang, J.L. Lei, A.G. Burns, M. Wiltberger, A.D. Richmond, S.C. Solomon, T.L. Killeen, E.R. Talaat, D.N. Anderson, Ionospheric electric field variations during a geomagnetic storm simulated by a coupled magnetosphere ionosphere thermosphere (CMIT) model. Geophys. Res. Lett. 35, L18105 (2008)

M. Wiltberger, W. Wang, A.G. Burns, S.C. Solomon, J.G. Lyon, C.C. Goodrich, Initial results from the coupled magnetosphere ionosphere thermosphere model: magnetospheric and ionospheric responses. J. Atmos. Sol.-Terr. Phys. 66, 1411-1423 (2004)

M. Wiltberger, R.S. Weigel, W. Lotko, J.A. Fedder, Modeling seasonal variations of auroral particle precipitation in a global-scale magnetosphere-ionosphere simulation. J. Geophys. Res. 114, A01204 (2009)

M. Wiltberger, E.J. Rigler, V. Merkin, J.G. Lyon, Structure of high latitude currents in global magnetosphereionosphere models. Space Sci. Rev. (2016). doi:10.1007/s11214-016-0271-2

B. Zhang, W. Lotko, O. Brambles, M. Wiltberger, J.G. Lyon, Electron precipitation models in global magnetosphere simulations. J. Geophys. Res. Space Phys. 120, 1035-1056 (2015)

B. Zieger, J. Vogt, K.-H. Glassmeier, T.I. Gombosi, Magnetohydrodynamic simulation of an equatorial dipolar magnetosphere. J. Geophys. Res. 109, A07205 (2004)

B. Zieger, J. Vogt, K.-H. Glassmeier, Scaling relations in the paleomagnetosphere derived from MHD simulations. J. Geophys. Res. 111(A6), A06203 (2006a)

B. Zieger, J. Vogt, A.J. Ridley, K.H. Glassmeier, A parametric study of magnetosphere-ionosphere coupling in the paleomagnetosphere. Adv. Space Res. 38, 1707-1712 (2006b) 